\title{
Modus des Intimen
}

$\mathrm{Zu}$ Narrativität und Materialität im zeitgenössischen Experimentalfilm am Beispiel von Martin Arnolds Pièce Touchée

Le mode de l'intimité. Narrativité et matérialité dans le cinéma expérimental contemporain : Pièce Touchée de Martin Arnold

The Mode of Intimacy. On Narrativity and Materiality in Contemporary

Experimental Cinema Based on Martin Arnold's Pièce Touchée

\section{Svetlana CHERNYSHOVA M.A.}

\section{(2) OpenEdition}

\section{Journals}

Édition électronique

URL : http://journals.openedition.org/ceg/3918

DOI : 10.4000/ceg.3918

ISSN : 2605-8359

\section{Éditeur}

Presses Universitaires de Provence

Édition imprimée

Date de publication : 15 novembre 2018

Pagination : 173-184

ISBN : 979-10-320-0183-7

ISSN : 0751-4239

\section{Référence électronique}

Svetlana CHERNYSHOVA M.A., « Modus des Intimen », Cahiers d'Études Germaniques [Online], 75 |

2018, Online erschienen am: 25 April 2020, abgerufen am 28 November 2020. URL : http://

journals.openedition.org/ceg/3918; DOI : https://doi.org/10.4000/ceg.3918 


\section{Modus des Intimen}

\section{Zu Narrativität und Materialität im zeitgenössischen Experimentalfilm am Beispiel von Martin Arnolds Pièce Touchée}

Svetlana CHERNYSHOVA M.A.

Heinrich-Heine-Universität Düsseldorf

\section{Narrativität und Materialität ${ }^{1}$}

Die Begriffe Narrativität und Materialität finden immer mehr Einzug in interdisziplinäre Diskursräume. Doch bei einer definitorischen Annäherung breitet sich schnell ein Unbehagen aus, welches nicht zuletzt mit dem Status der beiden Begriffe zusammenhängt - dem Status des Nicht-Konturierten. Der vorliegende Beitrag sieht seine Aufgabe somit in dem Versuch, die beiden Begrifflichkeiten sowie deren Relationen zueinander zu befragen, indem ein dritter Terminus - die Intimität - mit in den Fokus der Auseinandersetzung gerückt wird.

Unter Narrativität kann, allgemein gesprochen, eine Bündelung oder Versammlung von Praktiken verstanden werden, die den Akt des Erzählens in der spezifischen Form ,als‘ Erzählung hervorbringt. Narrativität impliziert damit alle Rahmungen, Strukturen und Vorgänge, die eine Erzählung als eine solche bedingen. Doch auch diese Definition kann stark variieren, denn je nach unterschiedlichen disziplinären Positionierungen - in Abhängigkeit davon, ob der Fokus auf das Erzählte oder das Erzählen gelenkt wird - divergieren die Terminusbestimmungen stark. ${ }^{2}$

Auch Materialität findet als Begriff ambige Beschreibungen, die sich in unterschiedliche Richtungen ausbreiten. Zum einen verweist Materialität auf physische Präsenzen, die sich auch im unmittelbaren Bezug zu Materie und

1. Der Beitrag basiert auf dem im Rahmen der Tagung „Narrativität und Materialität“ (24. März 2017, Université Sorbonne Nouvelle / CEREG) präsentierten Vortrag „Materialität und Intimität. Zu Narrativen im zeitgenössischen Experimentalfilm“.

2. Vera Nünning, „Narrativität als interdisziplinäre Schlüsselkategorie“, Auszug aus dem Jahresbericht „Marsilius Kolleg 2011/2012“, [http://www.marsilius-kolleg.uni-heidelberg. $\mathrm{de} / \mathrm{md} /$ einrichtungen/mk/publikationen/mk_jb_05_narrativitaet_als_interdisziplinaere_ schluesselkategorie.pdf], Stand: 10. August 2017. 
Material widerspiegeln, denn auch auf der Ebene des Assoziativen schwingt hier das Versprechen einer Stofflichkeit und ,Verkörperlichung“ (embodiment) mit. Zum anderen eröffnet Materialität aber auch die Möglichkeit eines grundlegenden Verständnisses des Relationalen, denn was es impliziert, ist ein ,ökologisches Denken $^{3}$, welches nicht (nur) nach Entitäten fragt, sondern auch nach deren Umgebungen, Verbindungen und gegenseitigen Stabilisierungen.

Die angeführten Überlegungen deuten zunächst eine Lesart an, die als eine dialektische aufgefasst werden könnte - d.h. Narrativität als etwas, was in erster Linie auf eine Erzählbarkeit hinweist und sich somit auf der Ebene von Bedeutungsproduktion und Kausalität bewegt, und Materialität, dagegen, als etwas, was primär die physische Beschaffenheit von Entitäten angeht und die Ebene der Sinnproduktion und Bedeutung nicht zwangsläufig tangiert. Somit würden die beiden Begriffe Sphären beschreiben, die per se voneinander abgegrenzt werden, was aber, auch im Kontext des vorliegenden Bandes, nicht ganz unproblematisch ist und fernerhin auch fruchtbare Denkrelationen ausschließt.

Der hier präsentierte Beitrag möchte diesem dialektischen Verhältnis entkommen und eine Denkrichtung aktivieren, die es zulässt, die beiden Begrifflichkeiten im Sinne einer „mutual inclusion“4 ${ }^{4} \mathrm{zu}$ begreifen, d.h. einem Verhältnis einer wechselseitigen Einwirkung, die nicht von a priori festgesetzten, ,fertigen' Entitäten ausgeht, sondern die prozessuale Hervorbringung betont. ${ }^{5}$

Um dieses spezifische Verhältnis, welches im Laufe des Beitrages als ein ,intimes“ herausgearbeitet werden soll, auszuhandeln, bietet die Arbeit Pièce Touchée $^{6}$ des österreichischen Experimentalfilmemachers Martin Arnold einen Umgang bzw. eine Auseinandersetzung mit Narrativitäts-MaterialitätsRelationen an, die sich dieser Frage auf eine affektiv-ästhetische Weise nähert und somit einen besonderen Zugang ermöglicht.

\section{Pièce Touchée}

Martin Arnolds Pièce Touchée (franz. das angefasste, berührte Stück) aus dem Jahr 1989 (s/w - 16mm) ist ein filmisches Montagestück, in dem eine

3. Siehe weitere Ausführungen zum Ökologie-Begriff bei Jane Bennett (Jane Bennett, Vibrant Matter: A Political Ecology of Things, Durham, Duke University Press, 2010), Karen Barad (Karen Barad, Agentieller Realismus. Über die Bedeutung materiell-diskursiver Praktiken, Berlin, Suhrkamp, 2012) und Tim Ingold (Tim Ingold, “Toward an Ecology of Materials", The Annual Review of Anthropology, 2012, [http://www.annualreviews.org/doi/pdf/10.1146/annurevanthro-081309-145920], Stand: 09. Juli 2017, S. 432.

4. Brian Massumi, What Animals Teach Us about Politics, Durham, Duke University Press, 2014, S. 4.

5. Siehe hierzu Hans Ulrich Gumbrechts Überlegungen zur „Oszillation zwischen Präsenzeffekten und Bedeutungseffekten“ (Hans Ulrich Gumbrecht, Präsenz, Berlin, Suhrkamp, 2012, S. 341).

6. Die Arbeit ist auf der Internetseite des Künstlers [www.martinarnold.info] sowie auf dem Videoportal YouTube abrufbar, Stand: 11. Februar 2018. 
18 Sekunden lange Found-Footage ${ }^{7}$-Filmszene, d.h. eine aus vorgefundenem Material entstandene Szene, von ihrer Dauer her um das ca. 50-fache ausgedehnt und Trick- bzw. Montagetechniken ausgesetzt wird. Zudem wird die Audiospur, die ,ursprünglich` einen Dialog beinhaltete, durch eine rhythmisierte Geräuschschleife ersetzt.

Auf der Ebene der Handlung lässt sich die Szene in wenigen Sätzen beschreiben: Eine junge Frau sitzt in einem Zimmer, eine Zeitschrift lesend, und scheint in Erwartung von jemandem zu sein. Eine kurze Zeit darauf betritt ein Mann die Wohnung und begrüßt die Frau mit sich andeutender Vertrautheit. Ein Begrüßungskuss, eine freundlich gestimmte Unterhaltung und ein leichtes Sich-Bewegen im Raum schreiben die Erzählung auf der visuellen Ebene fort und lassen diese zum Schluss hin abrupt enden.

Die in der Normalsicht mit einer statischen Kamera gefilmte Szene zeichnet sich durch einen harten Schwarz-Weiß-Kontrast aus und wechselt zwischen der Halbnahaufnahme und der Halbtotalen. Doch an dem Punkt, an dem der Fokus auf das Erzähltempo sowie die Schnittfrequenz gelenkt wird, macht sich vor allem die Ebene der Materialität spürbar und lässt uns die Sprechebene wechseln - von der vorgefundenen Szene hin zu Arnolds Arbeit. Die stark frequentierte alternierende Montage sowie Match $\mathrm{Cuts}^{8}$ erzeugen eine dominierende Bildrhythmisierung. Einige Bilder werden dabei gedehnt, manche Bildabfolgen abrupt unterbrochen und in einen Loop bzw. Reloop ${ }^{9}$ gesetzt. Während von Beginn an auf der akustischen Ebene ein Rhythmus dominiert, verändert sich die temporale Verdichtung der Bilder phasenweise.

Nach den ersten zwölf Sekunden, in denen der Titel eingeblendet wird, setzt die Arbeit mit einem Bild ein, welches zunächst als ein medial gestörtes Stillbild wirkt. Die Kamera ist auf die Halbnahaufnahme eingestellt, in der Mitte des Bildes ist die oben bereits erwähnte Frau im Sessel fokussiert, mit einem Türrahmen und einem weiteren angedeuteten Raum im Hintergrund. Um die Frau herum sind Einrichtungsgegenstände - Wand- und Leselampen, Pflanzen, Tapetenbahnen, Möbelstücke - angeordnet, die die Figur rahmen und das Bild vor allem in der Vertikalen prägen. Ebenso vertikal ausgerichtet hält die Frau

7. Unter Found Footage wird, in Analogie zu objet trouvé der bildenden Kunst, vorgefundenes Material verstanden, welches dann im Kontext einer weiteren filmischen bzw. künstlerischen Arbeit in der einen oder anderen Weise verwendet wird. Weitere Auseinandersetzung mit dem Verhältnis materieller Aneignung siehe Christa Blümlinger (Christa Blümlinger, Kino aus zweiter Hand. Zur Ästhetik materieller Aneignung im Film und in der Medienkunst, Berlin, Vorwerk 8, 2009).

8. Unter Match Cut wird ein Schnitt verstanden, „bei dem die zwei Aufnahmen durch bildliche, tönende oder metaphorische Parallelen verbunden sind“ (James Monaco, Hans-Michael Bock, Film verstehen. Das Lexikon, Hamburg, Rowohlt, 2011, S. 152).

9. Von einem Loop kann gesprochen werden, wenn Sequenzen in eine Dauerschleife gesetzt werden, die zu einer ,endlosen“ Wiederholung führt. Ein Reloop bezeichnet dabei eine ,rückwärts` abgespielte Dauerschleife. Somit stellt sich an dieser Stelle auch die Frage, inwiefern bei der beschriebenen Materialarbeit von einer temporalen Gerichtetheit, d.h. von einer Chronologie gesprochen werden kann im Sinne eines ,vorwärts“ und ,rückwärts“, denn eben jene Kategorien finden in Arnolds Arbeit ebenfalls eine neue Zusammensetzung. 
eine Zeitschrift in der Hand, auf die ihr Blick gelenkt ist. Die Hand der Frau, die die Zeitschrift festhält, rückt dabei besonders in den Vordergrund, denn sie führt den Blick von der schwach kontrastierten, beinah leer wirkenden Papierfläche in das Bildgeschehen hinein. Das Bild scheint zunächst unbeweglich, doch nach und nach schärft sich die Wahrnehmung und stellt sich auf das Bild ein, sodass wir als Zuschauende minimale Bewegungsspuren bemerken. So ist es zunächst das Zucken des kleinen Fingers der lesenden Frau, welches die Bildrhythmisierung eröffnet. Die Bewegung verstärkt sich zwischendurch und wird schubweise, neben Zerrungen, Unterbrechungen, Dehnungen und Wiederholungen auch von Umkehrungen und Spiegelungen begleitet. Die Arbeit wird von harten, binären Bewegungen durchzogen - Aufstehen/ Hinsetzen, Links/ Rechtsdrehung, Türschließen/ Türöffnen usw. Die interrupten Bewegungen - auf und zu, an und aus - kommen im Verlauf der Arbeit in Fahrt, bis sie schließlich in den letzten vier Minuten des Films zu schwindelerregenden Zirkulationen führen. Und so entsteht beim Sich-Einlassen auf diese Bildverläufe die Frage, was durch diese - wie ich es hier nennen möchte - Aktivität des Materials eigentlich passiert. Um sich dieser Frage anzunähern, schlage ich deshalb eine Auseinandersetzung mit dem Begriff der Intimität vor, denn dieser - und das sei den folgenden Überlegungen vorangestellt - verweist auf einen Modus, der ästhetische Interruptionen und Interventionen bedingt und somit auch Narrativität erst möglich macht.

\section{Intimität/ Materialität}

Intimität scheint zunächst als Begriff einen Diskurs anzudeuten, der das Private und das Körperlich-Sexuelle umkreist. Doch in meiner Auseinandersetzung geht es eher darum, diesen Begriff als einen Modus fruchtbar zu machen, um Relationen beschreiben zu können. Assoziativ betrachtet mag es zunächst fernerliegen, in diesem Kontext von Intimität zu sprechen, doch was hier in den Fokus rückt, ist eine übergreifender angelegte Frage nach Relationen bzw. nach dem Intimen als einer bestimmten Form von Zusammenkunft. Intime Relationen werden von mir nicht als primär zwischenmenschliche Formen von Beziehungen gedacht, sondern gehen darüber hinaus und gestehen es allen Formen von Entitäten zu. Gemeint ist an dieser Stelle, dass sich zwischen Entitäten eine intensive und verdichtete Verbindung ereignen muss, damit sich diese als bedeutungstragend stabilisieren können. Intimität wird von mir folglich als ein Modus verstanden, der das Aushandeln und Hervorbringen von Relationen ermöglicht. Dabei sind es Relationen, die unterschiedliche Zeitlichkeiten sowie Existenzmodi überlagern. Von Intimität spreche ich also dann, wenn sich eine relationale Intensität ereignet, die aber auch Unsicherheiten produziert und auf diese Weise Interferenzen entstehen lässt. 
Im Band Das Zusammenspiel der Materialien in den Künsten ${ }^{10}$ prägt der schweizerische Literaturwissenschaftler Thomas Strässle den Begriff der Intermaterialität, den er weiter differenziert und in der Dreierkonstellation Materialinteraktion - Materialtransfer - Materialinterferenz denkt. Unter Materialinteraktion versteht Strässle heterogene Verhaltensweisen von Materialien wie z.B. in Collagearbeiten. Mit Materialtransfer verbindet der Literaturwissenschaftler den Modus der Täuschung, wie z.B. beim Kautschuk, welcher visuelle Strukturen von Holz nachahmt. Der dritte Modus, die Materialinterferenz, beschreibt einen Prozess, bei dem etwas Drittes entsteht, wie z.B. eine Lösung von Salz und Wasser. Nach dieser Unterscheidung wird folglich denkbar, dass wir Arnolds Arbeit als eine intermaterielle betrachten können. Zumindest scheinen die Modi der Materialinteraktion sowie der Materialinterferenz auch für Pièce Touchée anwendbar zu sein und markieren auf diese Weise die Materialitätsprozesse. Auf der Ebene der Materialität macht sich somit eine Spannung auf, denn das Material - das Ursprungsmaterial - wird vervielfältigt, wiederholt sich also selbst, wird in dieser Wiederholung aber in der Neukonstellation wieder gebrochen und negiert. Die Spannung an dieser Stelle besteht folglich darin, dass das Material durch die Wiederholung und Auflösung aufs Neue Materialität kreiert bzw. vielmehr diese steigert und überhaupt erst wahrnehmbar macht. Das Verhältnis des Materials zu sich selbst spaltet sich also auf eine paradoxe Weise auf, und lässt durch die Wiederholung, die gleichzeitig Differenzen produziert, Materialinterferenzen entstehen. Die Arbeit, die wir vor uns haben, ist etwas Anderes, etwas Drittes, was aber in verfremdeter Form auf das eigene Material verweist und paradoxerweise im gleichen Zug sich selbst zu markieren und zu demarkieren versucht. ${ }^{11}$

\section{Referenz}

Die materielle ,Ausgangsebene ${ }^{12}$ von Pièce Touchée bietet eine 18 Sekunden lange Filmszene aus dem US-amerikanischen Krimistreifen The Human Jungle ${ }^{13}$ aus dem Jahr 1954. Doch dieser formale ,Ursprung“ wird in den Arbeiten des Künstlers in seiner Bedeutung transformiert. Bei Arnold, als einem Künstler,

10. Thomas Strässle, Christoph Kleinschmidt, Johanne Mohs (Hrsg.), Das Zusammenspiel der Materialien in den Künsten. Theorien - Praktiken - Perspektiven, Bielefeld, transcript, 2013.

11. Siehe hierzu Gilles Deleuzes Auseinandersetzung mit Differenz und Wiederholung (Gilles Deleuze, Differenz und Wiederholung, Paderborn, Wilhelm Fink, 2007).

12. Der Begriff wird in Anführungszeichen gesetzt, um erneut zu markieren, dass es sich hierbei um kein lineares Verhältnis mit einem Anfangs- und Endpunkt handelt, weshalb die Begrifflichkeiten ,Ausgangsebene‘, ,Ursprung“, ,Original‘ usw. problematisiert gedacht werden müssen.

13. Der Film des Regisseurs Joseph M. Newman, der sich seinem Genre nach zu ,Film Noir‘ einordnen lässt, erzählt die Geschichte eines Polizisten, der in einem Mord an einer Striptease-Tänzerin ermittelt. Gerahmt wird die Handlung vom Umstand, dass der Polizist seiner Frau gegenüber verspricht, nur vorübergehend in die gefährlichen Ermittlungen verwickelt zu werden, doch im Laufe der immer komplexer werdenden Handlung entscheidet er sich, seiner Berufung trotz der Gefahr weiter nachzugehen. 
der primär mit Found-Footage arbeitet, also mit vorgefundenen medialen Produkten, scheint die Frage nach dem Umgang mit dem sog. Original eine ambivalente. So bringt diese auch einen Zwiespalt mit sich - die auf knapp 16 Minuten ausgedehnte Filmszene hat zum einen eine autonome Eigenständigkeit, da die Arbeit es nicht verlangt, den originären Filmausschnitt zu kennen und eindeutig verorten zu können, zum anderen kann und will sich Pièce Touchée aber auch nicht ganz von der Referenzialität freisprechen ${ }^{14}$. Auf dieser Ebene nimmt das vorgefundene Material mitunter auch die Funktion eines Verweises auf - Arnolds Arbeit nutzt den Film als Referenz, als Vorlage. Auf dieser Ebene wird eine kontextuelle Konturierung also mitgedacht, denn selbst ohne fundierte Kenntnisse der Filmgeschichte lässt sich der Ausschnitt in etwa in den 50-er Jahren des vorigen Jahrhunderts verorten.

Auch auf der auditiven Ebene macht sich dieses spezifische Materialitätsverhältnis bemerkbar. Der Film setzt mit einem extradiegetischen, phonetischen Einbruch als einem Off-Screen-Sound ein. Noch bevor wir uns als Zuschauende auf das Bild einlassen können, ist der mechanistische Rhythmus bereits da. Als Index einer omnipräsenten, aber nicht visuell greifbaren Apparatur durchzieht das taktgebende Geräusch den gesamten Film. Dabei ist der Rhythmus, bis auf eine minimale, kurz ansetzende Beschleunigung, gleichbleibend. Das Geräusch wiederholt sich in einer Schleife von nur wenigen Sekunden und gibt einen festen Takt an. Was ruft dieses mechanische Geräusch hervor? Auch wenn es weder benannt noch in irgendeiner Form visuell dargestellt wird, liegt die Vermutung sehr nahe, dass es sich um Geräusche eines Filmapparates handelt, die beim Durchlaufen eines Filmstreifens durch die Apparatur entstehen und nur gehört werden könnten, wenn man sich in der Nähe des Gerätes befände. Es sind Geräusche, die einem Filmvorführer vertraut sind, aber immer schon von der filmischen Erfahrung, von der Erfahrung im Kino abstrahiert werden. Was hier also stattfindet, ist das Intensivieren einer auditiven Materialerfahrung. Wir hören die Maschine, wir hören das Material, wir erfahren die Vorgänge des physischen Durchlaufens eines Filmstreifens - dennoch aber schon immer medial vermittelt. Fernerhin kommt es zu einem weiteren Unsicherheitsmoment, zu einer Verschiebung, und zwar auf der Ebene zwischen Auditivem und Visuellem. Auch hier ist das Verhältnis ein ambivalentes. Zum einen isolieren sich die beiden Ebenen voneinander, denn es wird eindeutig spürbar, dass die Geräusche nicht aus der Narration des Films entstammen und auch nicht damit korrespondieren. D.h. die Audiospur scheint eigenständig für sich zu laufen, auf der anderen Seite wird aber gleichzeitig deutlich, dass die Grenzlinie porös ist und ganz andere Korrelationen aufzeigt. Das mechanische Geräusch gibt den Takt an, nachdem sich die Schnittreihenfolge der Bilder ordnet, was in der filmischen Fließbewegung dazu führt, dass die Filmfiguren, mit all ihren Zuckungen,

14. Die von Arnold ,bearbeitete‘ Szene zeigt die wartende Frau sowie die kurz darauf stattfindende Begrüßung zwischen der Frau und ihrem Mann, dem Protagonisten, in ihrem gemeinsamen Zuhause, nachdem der Mann zur Tür hereintritt. 
Rupturen und Verzögerungen, sich mit dem Rhythmus des Apparates bewegen. Was also auf der Ebene der Audio-Video-Relation aufklafft, ist die Erzählbarkeit der Materialität. Das Filmmaterial, die einzelnen Schnitte und Sequenzverfremdungen werden selbst zu Aktanten und prägen die Inszenierung mit. Somit verwendet die Arbeit bestimmte filmische Narrative, um diese aber schließlich zu rematerialisieren und eine Werkerzählbarkeit aufzubauen, die sich der gewohnten filmischen Linearität entzieht. So scheint das Narrative eine Präsenz zu haben, die sich aber einem direkten Zugriff verweigert. Das Narrativ besteht in einer Latenz, einem Verweis, der die Arbeit im gleichen Zug konstituiert, aber auch die eigene Auflösung, den eigenēn Aufbruch zulässt bzw. vorantreibt.

\section{Subjektivität}

Wenn wir uns mit Intimität auseinandersetzen, drängt sich zwangsläufig eine weitere Frage auf - und zwar handelt es sich hierbei um die Frage nach dem Subjekt bzw. nach der Subjektivität. Ausgehend von der Alltagsvorstellung, Intimität sei per se subjektgebunden zu denken, möchte ich diese Annahme problematisieren, und, wie bereits weiter oben angeführt, in einen Denkraum überführen, der nicht subjektzentriert ist.

Wie bereits angesprochen, provoziert Pièce Touchée eine Infragestellung des Narrativs. Durch die Ambivalenz lässt die Arbeit nicht zu, dass wir uns als Zuschauende eindeutig positionieren und eine abgrenzbare, stabile Narration benennen können. In jedem Moment des Greifen-Wollens scheint das Feste wieder zu kippen. Die Arbeit kreiert folglich einen Modus, den ich als einen intimen bezeichne, denn es ist eine Position, die sich vor allem durch Momente von Nähe, die sich aber als zu unsicher entpuppen, kennzeichnet. Pièce Touchée bringt auf diese Weise, durch die Intimität, eine Subjektivität hervor, die nicht stabil sein kann und einen permanenten Suchmodus initiiert. Hier produziert die Intimität „flimmernde Subjekte“ ${ }^{15}$, die sich dem Fixierbaren entziehen. Als Zuschauerin weiß ich nicht, welcher Narration ich folgen soll, was zur Folge hat, dass ich keine souveräne Position annehmen kann. Ich verbleibe in der Ambiguität bzw. werde erst in der Ambiguität konstituiert. So ist es die Intimität, die mich als ein flimmerndes Subjekt hervorbringt. Erst das Einlassen auf die Arbeit, das Mitgenommen-Werden durch das Material schafft es letztendlich, dass Subjektivität denkbar sein kann. Was ich als Intimität bezeichne, ist jener Modus, der die Momente erst möglich macht, in denen sich eine Intervention und Intensität ereignen können.

Wenn wir Subjektivität hier folglich als etwas begreifen, was aus den Prozessen der intimen Materialarbeit resultiert, dann erscheint es sinnvoll oder vielmehr notwendig, unsere Aufmerksamkeit erneut auf die ,Eigenaktivität des Materials‘ zu richten. D.h. das System, in dem wir uns befinden, ist

15. Der Begriff des ,flimmernden Subjekts“ ist im Rahmen eines sehr produktiven Gesprächs mit Prof. Timo Skrandies entstanden. 
nicht subjektzentriert und versucht nicht von bereits bestehenden Entitäten auszugehen, sondern gerade die Involviertheit zu betonen, die in Milieus ${ }^{16}$ denkt und Materialität also auch als etwas begreift, was aktiv ist. Was wir hier Subjekt nennen können, ist schlicht ein mögliches Resultat von diesen Prozessen, die ich als intime bezeichne. Es sind Prozesse, die Intensitäten voraussetzen und erzeugen, aber gleichzeitig auch Momente des Unsicheren produzieren und keine Entscheidbarkeit ermöglichen.

\section{Intimität und Gewohnheit}

Wenn wir Intimität nun als etwas begreifen, was das Aushandeln von Situationen und Positionen bedingt bzw. eine Konstituierung und Stabilisierung erst ermöglicht, hängt mit dieser Überlegung gleichzeitig eine Vorstellung zusammen, die von einem Momentum des Gewohnten ausgeht. Intimität ermöglicht folglich ein Umgehen mit gewohnten Verhältnissen und lässt sich als eine Intervention, eine Verschiebung begreifen, denn sie lässt das Alltägliche, das Routiniert-Inkorporierte als instabil erfahren. Das Gewohnte bezeichnet somit bereits bestehende Relationen, die in der intimen Intensivierung auf eine radikale Art und Weise in Frage gestellt werden, um im gleichen Zug eine ReKonfigurierung zu ermöglichen, jedoch, ohne dass diese Konfigurierung von unhintergehbaren Stabilitäten ausgeht. Intimität ermöglicht ein spezifisches Heraustreten aus Verhältnissen, doch diese Verhältnisse werden nicht als ,vorher bereits festgelegte, sondern als just in diesem Moment als greifbare erfahren.

Was die Intensität von Pièce Touchée, dieser Lesart nach, ausmacht, ist das Verweisen auf das Gewohnte, welches sich erst im Entzug dessen erfahrbar macht. Die Bewegungen, die hier als ,Ausgangsebene“ bzw. Referenz verwendet werden, adressieren zum einen das Gewohnte - das Hinsetzen und Aufstehen, das Öffnen und Schließen von Türen usw. - gleichzeitig produzieren sie dabei aber auch etwas Anderes, was die gewohnten Strukturen als grotesk erscheinen lässt. In einer knapp eine Minute lang dauernden Szene (8:10-9:15) ereignet sich beispielsweise etwas, was als ein Kussmotiv beschrieben werden kann: Die Frau sitzt, in der Halbnahen mittig im Bild platziert, in ihrem Sessel und schaut hinauf zu dem zu ihrer rechten Seite stehenden Mann, der sich leicht über den Sessel beugt und den Blick der Frau erwidert. $\mathrm{Zu}$ der Linken der Frau befindet sich eine mit einem großen Schirm bestückte Leselampe, die die Figur des Mannes im Bild symmetrisierend ausgleicht. Hinter dem Paar deutet sich ein weiterer Raum an, der, mittig fokussiert, die vertikale Ebene des Bildes prägt. Nach ca. 20 Sekunden wird das Bild entlang der mittigen Vertikalen gespiegelt und changiert dann im Verlaufe der nächsten 40 Sekunden seine Links-Rechts-Ausrichtung. Das Tempo variiert dabei so stark, dass die Konturen der jeweiligen Figuren bzw.

16. Dem Philosophen Gilles Deleuze folgend, vereint der Milieubegriff, in Anlehnung an das französische Wort, sowohl die ,Mitte“ als auch die ,Umgebung“ bzw. das „Habitat“ (Isabelle Stengers, „Introductory Notes on an Ecology of Practices“, [http://epress.lib.uts.edu.au/journals/ index.php/csrj/article/view/3459/3597], Stand: 10. August 2017, S. 187). 
Gegenstände einige Sekunden lang nicht mehr klar abgrenzbar wahrgenommen werden können und ein Wechselspiel erzeugen, bei dem die Bildbewegung die narrativ-immanenten Bewegungen übersteigt.

Die Bewegungen, die sich immer wieder andeuten und sich der Gewohnheit und Erfahrung nach als ein Kuss entfalten müssten, werden in der Szene immer wieder unterbrochen. Während das Ansetzen zum Kuss zunächst ganz langsam beginnt, werden der Bildwechsel und die visuell greifbaren Bewegungen immer deutlicher und präsenter, bis der Rhythmus durch eine zusätzliche Spiegelung des Bildes zu einer exzessiven Steigerung führt und seinen Höhepunkt erreicht. Hier verliert das Visuelle für einen kurzen Augenblick seine Kontur und wird zu einem verschwommenen, überlagerten Ding. Durch den gesteigerten Rhythmus bekommen die Bewegungen etwas Obszönes ${ }^{17}$, da die Gesten durch die Andeutungen immer wieder aufs Neue einen Überschuss an Bedeutung produzieren, die aber im Moment ihrer eigentlichen Entfaltung auf die radikalste Art und Weise unterbrochen werden.

Wenn wir uns an dieser Stelle erneut dem Begriff des Narrativs zuwenden und das Verhältnis zwischen dem Ursprungsmaterial und Arnolds Arbeit anschauen, wird hier eine Unsicherheit spürbar, denn der Kuss verbleibt in der Andeutung. Von der Narration her ist der Kuss in der Latenz da, doch die eigentliche Berührung wird zusammengerafft überspielt. Der Kuss entpuppt sich als eine verkrampfte Geste, die in und durch die Wiederholung und Entfaltung ihre eigene Leere markiert. So prägt die Philosophin Lauren Berlant in ihrem Buch Cruel Optimism ${ }^{18}$ den Begriff ,Good Life-Genre Damit beschreibt sie Lebensnarrative, die zwar stark affektiv aufgeladen sind und, als gesetzte Formen, Stabilität und Halt versprechen, dennoch aber nicht über die bloße Form hinausgehen und dem Subjekt immer wieder aufs Neue die eigene Unzulänglichkeit und Unfähigkeit demonstrieren, sich auf relationale Konfigurationen einzulassen, die eine tatsächliche Ansprache und Offenheit zulassen. Nach Berlant bewegen wir uns in diesem Good-Life-Modus und laufen Zuständen hinterher, die sich als nicht haltbare, als ,grausame‘ erweisen. Auf den Moment der unbeabsichtigten Wiederholung geht auch Sigmund Freud in seiner Auseinandersetzung mit dem Unheimlichen ${ }^{19}$ ein. Eben jene - unbeabsichtigte - Wiederholung beschreibt er als unheimlich, denn was dabei entstehe, sei das Empfinden von etwas Verhängnisvollem und Unentrinnbarem, welches aber Bewegungen innewohne, die für uns zutiefst heimlich und nah seien. Was diese Wiederholung folglich produziert, ist eine Spaltung, die auf das Gewohnte verweist und in der gleichen Geste das Fremde als etwas entpuppen lässt, was bereits die intime Nähe zu uns hat und eben dadurch als unheimlich und bedrohlich erfahren wird. So zeigen sich die durch die Materialarbeit entstandenen Gesten in Martin Arnolds Pièce Touchée als abrupt und pathologisch wiederholt. Die Rematerialisierung der

17. Jean Baudrillard, Passwörter, Berlin, Merve, 2002, S. 27ff.

18. Lauren Berlant, Cruel Optimism, Durham, Duke University Press, 2011.

19. Sigmund Freud, „Kleine Schriften II“, 1919, projekt.gutenberg.de, [http://gutenberg.spiegel.de/ buch/kleine-schriften-ii-7122/29], Stand: 10. Juni 2017. 
Narrative und die Intensivierung des Materials führen hier dazu, dass die Bilder etwas Unheimliches produzieren.

Was in Arnolds Arbeit passiert, ist das ambivalente Selbst-Intensivieren von Materialität. Das Material - der physisch greifbare Film - wird durch die Transformations- und Überlagerungsschritte zu einem gesteigerten Medium, welches sich, Bild für Bild, der Kontrolle entzieht und gerade diese Widerspenstigkeit markiert. Die Schnitte, Raffungen, Dehnungen, Wiederholungsloops, Spiegelungen, Umkehrungen, Verzögerungen, Beschleunigungen führen gerade dazu, dass das Material als Material spürbar wird. Während eine Abfolge von Bildern, die, pauschal formuliert, unserer filmalltäglichen Gewohnheit entspricht, also einen visuell-kausalen Verlauf aufweist, dazu führt, dass die Ebene der Materialität in den Hintergrund rückt, wird diese Ebene hier gerade an den Punkten des Ausschweifens, durch die Bruchstellen und Momente der Verzögerungen markiert.

Während das ursprüngliche Material - der Kriminalfilm aus dem Jahr 1954 - bestimmte Beziehungsnarrative andeutet - das Paar, die Frau als die Wartende - zeigt die Arbeit gerade die Diskontinuitäten des Erzählgestus auf. Durch das Entnehmen einer Szene und das agentiale ${ }^{20}$ Neuversammeln von einzelnen Bildern (Spiegelung, Umkehrung, Zerrung usw.) löscht die Arbeit die vermuteten Narrative aus, markiert sie auf diese Weise aber gleichzeitig und produziert somit auch neue Narrationen. Zum einen bleibt das Narrativ also spürbar, wird andererseits aber seiner kausalen Logik entzogen und durch die Materialzerrungen zu etwas Anderem transformiert.

\section{Schlussbemerkungen}

Was ich in meinem Beitrag versucht habe zu verdeutlichen, ist der Gedanke, dass die Materialität bzw. Arnolds Arbeit Pièce Touchée als Agency etwas entstehen lässt, was wir als intim bezeichnen können. Durch die Überlagerungen des Materials, die Materialinteraktionen und -interferenzen, konnte eine Narrativität entstehen, die nicht in Eindeutigkeit aufgehen kann. Pièce Touchée produziert eine Intimität, weil die Arbeit etwas spürbar macht, was sich ohne diese Intensivierung des Materials nicht zeigt. Das Bedrohliche des Kusses, das Zuckend-Verkrampfte der Umarmung, das Obszön-Abgeschnittene der Geste bringt etwas hervor, was den Bewegungen inhärent zu sein scheint, sich aber erst in der Verschiebung von Zeitlichkeiten, erst in der Arbeit im Material, erst in der Steigerung dessen zeigt. Der Kuss wird erst intim, weil plötzlich die pathologischen Unterbrechungen und Wiederholungen spürbar werden, weil jede Annäherung auch Scheitern impliziert. Diese Überlegung führt dazu, dass sich auch Subjektivität als eine mögliche Existenzform begreift und schon immer auch erst als Resultat erfahren

20. Siehe weitere Ausführungen zum Agency-Begriff bei Karen Barad (Karen Barad, Agentieller Realismus. Über die Bedeutung materiell-diskursiver Praktiken, Berlin, Suhrkamp, 2012) und Bruno Latour (Bruno Latour, Eine neue Soziologie für eine neue Gesellschaft. Einführung in die Akteur-Netzwerk-Theorie, Suhrkamp, Frankfurt a.M., 2007). 
werden kann. D.h. es ereignet sich bereits etwas, was sich erst in der Verschiebung als das Intime zeigt und eben in diesem Moment kann es zu dem ereignishaften Gestus kommen, den wir als Subjektivität bezeichnen können und der Narrativität erst möglich macht. Die mechanischen Rhythmisierungen, getakteten Schnitte, Wiederholungen und Umbrüche resultieren auch in einer starken körperlichen Ansprache - in der Irritation, im Schwindelgefühl. Hier ist es die Materialität, die sich auf dieser Ebene spürbar zeigt. Das unaufhörliche, penetrante Geräusch, die teils pathologisch wirkenden Bewegungen, das Zerstückelte, das einerseits Grotesk-Humorvolle und andererseits verstörend Wirkende in den abgehackten Bewegungen nimmt uns mit, konstituiert uns und sorgt dafür, dass diese Überlagerungen von Materialität eine Intimität erzeugen, die notwendig ist, um von Bedeutung, von Erzählbarkeit, von Narrativität sprechen zu können. Die Bewegungen sind teils obszön, teils herausfordernd und bringen etwas hervor, was sich zunächst nicht einordnen lässt. Es sind wiederholte Momente, die sich selbst wiederholen und in der Wiederholung auslöschen, sich neu hervorbringen und mit jedem Schnitt die Aufmerksamkeitsmuster prüfen und herausfordern. Die Wiederholung der ungewohnt-gewohnten Bewegungen wird zur Gewöhnung, um sich dann wieder erneut zu brechen. Immer wieder werden die bekannten Muster von den intensivierten Gesten heimgesucht. Als Zuschauende bleiben wir stets in Erwartung. 
